OPEN ACCESS

Edited by:

$X i$ Yang,

Fudan University, China

Reviewed by:

Qing Guo,

Jiangsu Taizhou People's Hospital,

China

Xiufeng Cao

Nanjing No. 1 Hospital, China

*Correspondence:

Wei-Guo Zhu

jshazwg@126.com

Yu-Suo Tong

tongyusuo@163.com

${ }^{+}$These authors have contributed equally to this work and share

first authorship

Specialty section: This article was submitted to Radiation Oncology, a section of the journal

Frontiers in Oncology

Received: 18 January 2021 Accepted: 24 June 2021

Published: 08 July 2021

Citation:

Xu Y-Y, Zhou X-L,

Yu C-H, Wang W-W, Ji F-Z,

He D-C, Zhu W-G and Tong Y-S (2021) Association of Sarcopenia

With Toxicity and Survival in Postoperative Recurrent Esophageal Squamous Cell Carcinoma Patients Receiving Chemoradiotherapy.

Front. Oncol. 11:655071. doi: 10.3389/fonc.2021.655071

\section{Association of Sarcopenia With Toxicity and Survival in Postoperative Recurrent Esophageal Squamous Cell Carcinoma Patients Receiving Chemoradiotherapy}

Ying-Ying $\mathrm{Xu}^{\dagger}{ }^{+}$Xi-Lei Zhou ${ }^{\dagger}$, Chang-Hua Yu, Wan-Wei Wang, Fu-Zhi Ji, Dong-Cheng He, Wei-Guo Zhu * and Yu-Suo Tong *

Department of Radiation Oncology, Huai'an First People's Hospital, Nanjing Medical University, Huai'an, China

Background: Sarcopenia has been associated with treatment-related toxicities and poor survival in cancer patients. Our aim was to investigate the prevalence of sarcopenia in postoperative recurrent esophageal squamous cell carcinoma (ESCC) patients receiving chemoradiotherapy (CRT) and evaluate associations with treatment-related toxicity and prognosis.

Methods: One hundred and eighty-four patients with postoperative locoregional recurrent ESCC receiving CRT between January 2014 and December 2016 were included. The skeletal muscle area (SMA) was measured at the third lumbar vertebra level. Sarcopenia was defined as skeletal muscle index $\left(\mathrm{SMI}=\mathrm{SMA} /\right.$ height $\left.^{2}\right)$ less than $47.24 / \mathrm{cm}^{2} / \mathrm{m}^{2}$ for men and $36.92 / \mathrm{cm}^{2} / \mathrm{m}^{2}$ for women. Association of sarcopenia with overall survival (OS) was analyzed using univariate and multivariate cox regression models.

Results: Sarcopenia was observed in 94 of 184 (51.1\%) patients. Sarcopenic patients had significantly higher rates of grade 3-4 toxicities compared to those without sarcopenia (36.2\% vs $21.1 \%, p=0.034$ ). The survival rate at 12 and 24 months was $36.2 \%$ and $3.2 \%$ in the sarcopenic patients and $57.8 \%$ and $17.8 \%$ in the non-sarcopenic patients $(p<$ 0.001). Multivariate cox regression analysis showed that sarcopenia was significantly associated with decreased OS (HR $=1.729,95 \% \mathrm{Cl} 1.231-2.428, p=0.002)$.

Conclusions: Sarcopenia is an independent indicator of poor survival in postoperative locoregional recurrent ESCC patients treated with CRT. Early nutritional interventions before treatment may improve the prognosis.

Keywords: sarcopenia, esophageal squamous cell carcinoma, prognosis, chemoradiotherapy, survival 


\section{INTRODUCTION}

Esophageal cancer (EC) is one of the most common cancers worldwide and esophageal squamous cell carcinoma (ESCC) accounts for about $70 \%$ of all cases (1). ESCC is highly prevalent in China, and approximate $90 \%$ of new cases are ESCC (2). Radical esophagectomy with two-field or three-field lymph node dissection is the primary treatment in locally advanced ESCC. However, the survival of patients treated with surgery alone is poor, with 5-year survival rates of only $25 \%-39 \%$ $(3,4)$. After surgery, $43 \%-53 \%$ of patients develop locoregional recurrence or distant metastasis (5). For these patients, palliative chemotherapy or chemoradiotherapy (CRT) are commonly used to control cancer-related symptoms and prolong survival. Although these approaches have been demonstrated to be effective in around $50 \%$ of patients (6), it is also associated with severe hematological and gastrointestinal toxicities. Thus, discovering factors that could predict CRT-related toxicity and survival in ESCC patients are urgent needs.

Sarcopenia is a common geriatric syndrome, which is initially defined by Baumgartner et al. to describe age-related loss of skeletal muscle mass and skeletal muscle strength (7). Age is the main cause, but not the sole cause of sarcopenia. Malnutrition, low levels of physical activity, several chronic diseases and cancer also induce sarcopenia (8). Many studies have demonstrated that systemic inflammation, inadequate energy and protein intake, as well as increased metabolic rate are independent risk factors for sarcopenia (9). These risk factors are prevalent in ESCC. In a recent study by Anandavadivelan $\mathrm{P}$. et al, sarcopenia and sarcopenic obesity was observed in $43 \%$ and $14 \%$ of EC patients, and the presence of sarcopenic obesity was a risk factor for developing dose limiting toxicity during neoadjuvant chemotherapy (10). Currently, skeletal muscle area (SMA) on abdomen CT imaging at the level of third lumbar vertebra is widely used for detection of sarcopenia, and it has been demonstrated as a reliable method for whole body muscle mass assessment (11). Sarcopenia is frequently seen in patients with advanced tumor. Prior studies have demonstrated that sarcopenia is associated with poorer overall survival (OS) for a number of malignancies, such as pancreatic cancer, renal cell carcinoma, colorectal cancer (12-14). In an Asian study of ESCC patients receiving neoadjuvant CRT, Ozawa $\mathrm{Y}$ et al. found that pretherapeutic sarcopenia was significantly correlated with treatment response and worse disease free survival (15). However, its impact on patients with recurrent or metastatic ESCC remains largely unknown. Thus far, only one study investigated sarcopenia in advanced esophagogastric cancer patients and reported that there was no association between sarcopenia and survival and treatment-related toxicity during palliative chemotherapy (16). However, that study comprised a limited number of patients (only 88 patients included) and the majority of patients were adenocarcinoma (83\%). The effect of sarcopenia remains unclear in patients with postoperative locoregional recurrent ESCC.

Therefore, the current study aimed to determine the incidence of sarcopenia in patients with postoperative locoregional recurrent ESCC and to evaluate the relationship between sarcopenia on treatment-related toxicity and OS in patients treated with CRT.

\section{MATERIALS AND METHODS}

\section{Patients and Study Design}

In this retrospective analysis, patient with ESCC who were treated at our institution from January 1, 2014, to December 31 , 2016, for locoregional recurrences after surgery were screened. This time period was chosen in order to have adequate follow-up time for analysis of OS. Postoperative recurrences were confirmed by biopsy, $\mathrm{CT}$, and/or positron emission tomography (PET)/-CT fusion scans. Eligible patients were less than 75 years old; Karnofsky performance status (KPS) score $\geq 70$; had histopathologically confirmed ESCC; had local postoperative recurrence (anastomotic recurrence and/or locoregional lymph node metastasis); had upper abdominal CT scan within 3 weeks of treatment start. Patients were excluded if they had incomplete resection, were treated with neoadjuvant or postoperative radiotherapy, had distant metastasis (other than metastasis to supraclavicular or celiac lymph node), or with severe comorbidities. The 7th edition of tumor-node-metastasis (TNM) classification for esophageal carcinoma (UICC, 2009) was used to stage the primary disease after surgery. This study was approved by the institutional review board of Huai'an First Hospital. Informed consent was exempted due to the retrospective nature of the study.

\section{Treatment Details}

Radiotherapy: Patients received either 3-dimensional conformal radiation therapy or intensity modulated radiation therapy. Treatment-planning CT scans (16-slice Philips Brilliance Big Bore CT) using intravenous contrast with a slice thickness of $5 \mathrm{~mm}$ were performed for all patients in the supine position. In the present study, all patients were treated with involved field radiotherapy. The gross tumor volume (GTV) included the recurrent tumor or the metastatic lymph nodes. The clinical target volume (CTV) was generated by using $0.8-1 \mathrm{~cm}$ radial margin and 1.5-2.0 cm longitudinal margins to the GTV. The planning target volume (PTV) was defined as the CTV plus a $0.5 \mathrm{~cm}$ margin in all directions. The prescribed dose was 50 to 66Gy (1.8-2.0 Gy/fraction, 5 days a week) for PTV.

Chemotherapy regimens: Most patients received docetaxel and cisplatin (DP) based chemotherapy in our institution. Among them, 71 patients received concurrent chemotherapy comprising docetaxel $\left(25 \mathrm{mg} / \mathrm{m}^{2}\right)$ and cisplatin $\left(25 \mathrm{mg} / \mathrm{m}^{2}\right)$ weekly for 5-6 weeks. Another 42 patients received docetaxel and cisplatin regimen consisted of $75 \mathrm{mg} / \mathrm{m}^{2}$ docetaxel on days 1 and 22 , and $25 \mathrm{mg} / \mathrm{m}^{2}$ cisplatin on days $1-3$ and 22-24. Approximately 4 to 5 weeks after completion of radiotherapy, at least 2 cycles of adjuvant chemotherapy (docetaxel $75 \mathrm{mg} / \mathrm{m}^{2}$ on day 1 , cisplatin $25 \mathrm{mg} / \mathrm{m}^{2}$ on days 1-3) were given to patients who still have sufficient performance status.

From January 2016, patients aged older than 70 years were treated with S-1 (70 mg/m², twice per day, on days 1-14 and days 
22-36) based concurrent chemotherapy to avoid the severe adverse events. In these patients, adjuvant chemotherapy with S-1 (70 mg/m², on days $1-21$ every 4 weeks) was done after radiotherapy if possible.

\section{Body Composition Analysis}

Regional muscle tissues were measured by the upper abdominal CT from electronically stored images, which has been done within 3 weeks of radiotherapy. The third lumbar vertebra (L3) was selected as the landmark. Two consecutive CT images extending from L3 were used to measure total muscle crosssectional area and the mean cross-sectional area $\left(\mathrm{cm}^{2}\right)$ was calculated from each patient. The total SMA including psoas, paraspinal and the abdominal wall muscles (Figure 1) were hand-drawn by a senior radiotherapy oncologist. Skeletal muscle was identified and quantified within a Hounsfield unit (HU) range of -29 to $+150 \mathrm{HU}$ (17) using the Monaco TPS software (Elekta). The mean cross-sectional areas were normalized to the square of body height $\left(\mathrm{cm}^{2} / \mathrm{m}^{2}\right)$ and presented as skeletal muscle index (SMI). Sarcopenia was defined as SMI less than

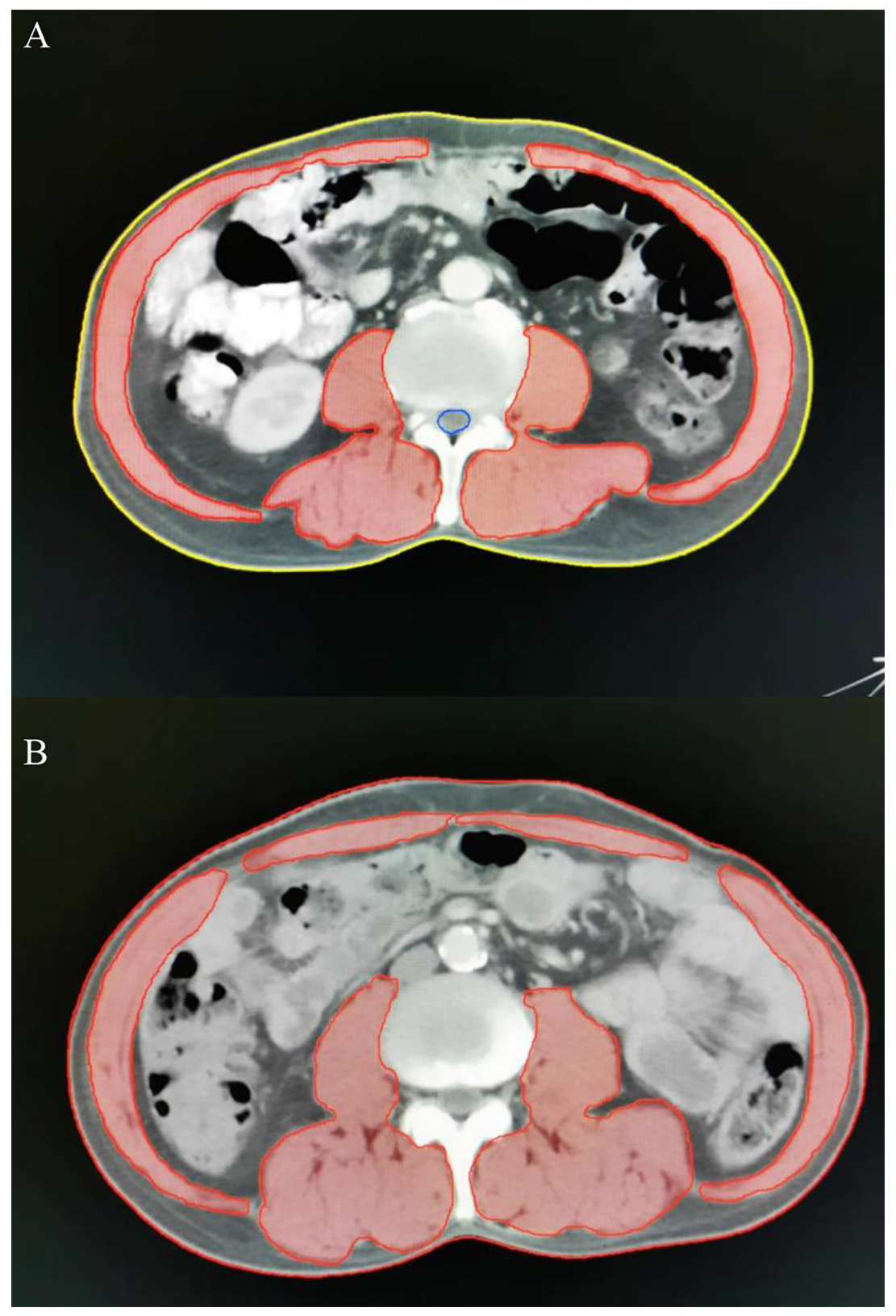

FIGURE 1 | Axial computed tomography (CT) images at the level of L3 with skeletal muscle highlighted in red. Representative CT images in patients with (A, a-67-year-old male patient, SMA $=122.85 \mathrm{~cm}^{2}$, BMl $\left.=21.38 \mathrm{~kg} / \mathrm{m}^{2}, \mathrm{SMl}=41.04 \mathrm{~cm}^{2} / \mathrm{m}^{2}\right)$ and without sarcopenia $(\mathbf{B}$, a-62-year-old male patient, SMA $=158.64$ $\left.\mathrm{cm}^{2}, \mathrm{BMI}=21.51 \mathrm{~kg} / \mathrm{m}^{2}, \mathrm{SMl}=56.88 \mathrm{~cm}^{2} / \mathrm{m}^{2}\right)$. SMA, skeletal muscle area; BMI, body mass index; SMl, skeletal muscle index. 
$47.24 / \mathrm{cm}^{2} / \mathrm{m}^{2}$ for men and $36.92 / \mathrm{cm}^{2} / \mathrm{m}^{2}$ for women, using previously published cut-off values associated survival in patients with EC (18).

\section{Patient Data}

The present study collected pre-treatment data including patient demographics, serum albumin, weight, height, and body mass index (BMI). The BMI was calculated as weight $(\mathrm{kg}) /$ height $\left(\mathrm{m}^{2}\right)$. Underweight, normal weight and overweight were defined as $\mathrm{BMI}<18.5,18.5-24.9$, and $\geq 25 \mathrm{~kg} / \mathrm{m}^{2}$, respectively (19).

\section{Toxicity Assessment and Follow-up}

Toxicity was classified according to the National Cancer Institute Common Toxicity Criteria for Adverse Events (NCI-CTCAE) version 4.0. In the first 2 years after treatment, patients were followed every 3 months, and every 4-6 months thereafter. The final data collection was May, 2020.

\section{Statistical Analysis}

Statistical analysis was performed using SPSS 20.0. Continuous variables are presented as median and range, and categorical variables are presented as number and percentage. For continuous variables, differences between groups were performed using Student's $t$ test or the Mann-Whitney U test. Categorical variables were compared using Chi-square test or Fisher's exact test. OS was calculated as the time from the last date of radiotherapy to the date of death due to any cause or last follow-up. Data from patients that had not died by the time of analysis were censored. The Kaplan-Meier method was used to determine effects of each variable on OS, and log-rank test was used to compare survival between groups. Univariate and multivariate cox proportional hazards regression models were used to identify prognostic factors of survival. Any factors with $p$ value less than 0.10 in the univariate analysis were included in the multivariate analysis. All $p$ values were two sided, and level of significance was $p$ less than 0.05 .

\section{RESULTS}

\section{Patient and Treatment Characteristics}

Between January 1, 2014, and December 31, 2016, 215 patients with postoperative locoregional recurrent ESCC who had received radiotherapy were retrospectively reviewed. Seventeen patients were excluded because the pretreatment CT images were not available for body composition analysis, 14 were excluded due to the lack of complete clinical data. Therefore, a total of 184 patients met inclusion criteria in the final analysis.

Baseline characteristics are displayed in Table 1. Of the 184 patients, $141(76.6 \%)$ were male, and the median age was 63 (range, 54-75 years). Almost $90 \%$ of patients (166/184) had KPS score $\geq 80$. Fifty-seven $(31.0 \%)$ patients had anastomotic recurrence +/- regional lymph node metastasis, and 127 (69.0\%) had only lymph node recurrence (supraclavicular in 28 , mediastinal in 39 , celiac in 14 , and multiregional lymph node metastasis in 46). The diagnosis of recurrence was based on
TABLE 1 | Patient and treatment characteristics.

\begin{tabular}{|c|c|}
\hline Characteristics & Number (\%) \\
\hline Age (year) median (range) & $62(51-75)$ \\
\hline \multicolumn{2}{|l|}{ Sex } \\
\hline Male & $141(76.6 \%)$ \\
\hline Female & $43(23.4 \%)$ \\
\hline \multicolumn{2}{|l|}{ KPS } \\
\hline$\geq 80$ & $166(90.2 \%)$ \\
\hline 70 & $18(9.8 \%)$ \\
\hline \multicolumn{2}{|l|}{ Primary tumor location } \\
\hline Upper third & $21(11.4 \%)$ \\
\hline Middle third & $124(67.4 \%)$ \\
\hline Lower third & $39(21.2 \%)$ \\
\hline \multicolumn{2}{|l|}{ Stage of primary tumor } \\
\hline Stage I-II & 77 (41.8\%) \\
\hline Stage III & $107(58.2 \%)$ \\
\hline \multicolumn{2}{|l|}{ Recurrence pattern } \\
\hline Anastomosis +/-regional lymph node & $57(31.0 \%)$ \\
\hline Regional lymph node & $127(69.0 \%)$ \\
\hline \multicolumn{2}{|l|}{ Treatment } \\
\hline Radiotherapy alone & 20 (10.9\%) \\
\hline CRT & $164(89.1 \%)$ \\
\hline Radiation dose (Gy) median (range) & $50.4(36-66)$ \\
\hline \multicolumn{2}{|l|}{ Concurrent chemotherapy regimen } \\
\hline Docetaxel + cisplatin & $113(61.4 \%)$ \\
\hline S-1 & $51(27.7 \%)$ \\
\hline \multicolumn{2}{|l|}{ Chemotherapy after radiotherapy } \\
\hline Yes & $157(85.3 \%)$ \\
\hline No & $27(14.7 \%)$ \\
\hline \multicolumn{2}{|l|}{ Weight loss in 6 months } \\
\hline$\geq 5 \%$ & $64(34.8 \%)$ \\
\hline$<5 \%$ & 120 (65.2\%) \\
\hline \multicolumn{2}{|l|}{$\mathrm{BMI}\left(\mathrm{kg} / \mathrm{m}^{2}\right)$} \\
\hline$<18.5$ & $36(19.6 \%)$ \\
\hline $18.5-24.9$ & $131(71.2 \%)$ \\
\hline$\geq 25$ & $17(9.2 \%)$ \\
\hline \multicolumn{2}{|l|}{ Albumin (g/L) } \\
\hline$<35$ & $32(17.4 \%)$ \\
\hline$\geq 35$ & $152(82.6 \%)$ \\
\hline \multicolumn{2}{|l|}{ Diabetes } \\
\hline Yes & $17(9.2 \%)$ \\
\hline No & $167(90.8 \%)$ \\
\hline $\mathrm{SMI}\left(\mathrm{cm}^{2} / \mathrm{m}^{2}\right)$ median (range) & $46.26(28.62-65.84)$ \\
\hline
\end{tabular}

KPS, karnofsky performance status; CRT, chemoradiotherapy; BMI, body mass index; $S M I$, skeletal muscle index.

biopsies in 64 patients (34.8\%). Follow-up CT and the clinical course were sufficient for diagnosis in the other 120 patients (65.2\%). The median time between CT scan and the start of radiotherapy was 7 days (range, 5-21 days).

All patients were treated with local radiotherapy, and only 4 patients $(2.2 \%)$ received less than $40 \mathrm{~Gy}$ because of treatmentrelated toxicities. Finally, 171 patients (92.9\%) completed radiotherapy as planned. The majority of patients (89.1\%, 164/ 184) were treated with concurrent chemotherapy, $113(61.4 \%)$ received concurrent DP and 51 (27.7\%) received S-1. Reasons for not received concurrent chemotherapy were KPS score $<80$ in 9 patients, refusal in 6 , and other reasons in 5 .

Before radiotherapy, approximately $34.8 \%$ of patients (64/ 184) had lost $\geq 5 \%$ of their body weight in the previous 6 months. The median BMI was $20.35 \mathrm{~kg} / \mathrm{m}^{2}$ (range, $15.26-27.40 \mathrm{~kg} / \mathrm{m}^{2}$ ), and 36 patients (19.6\%) were underweight. Overall, the median $\mathrm{SMI}$ in all cases was $46.26 \mathrm{~cm}^{2} / \mathrm{m}^{2}$ (range, 28.62-65.84 $\mathrm{cm}^{2} / \mathrm{m}^{2}$ ). 


\section{Prevalence of and Factors Associated With Sarcopenia}

Overall, sarcopenia was found in 94 patients (51.1\%). Patients with sarcopenia had worse KPS ( $p=0.024$, Table 2) and lower mean BMI ( $p<0.001$, Table 2$)$ than those without sarcopenia. Moreover, weight loss $\geq 5 \%$ in the previous 6 months $(46.8 \%$ versus $22.2 \%, p=0.001$, Table 2) and serum albumin $<35 \mathrm{~g} / \mathrm{L}$ (23.4\% vs $11.1 \%, p=0.033$, Table 2 ) were more frequently observed in the sarcopenic patients than in non-sarcopenia patients. With regard to tumor stage and patterns of recurrence, there was no significant difference between the two groups. Patients older than 70 years had a slightly higher prevalence of sarcopenia than those $<70$ years $(58.5 \%$ vs $47.1 \%, p=0.166$, Table 2), but the difference was not statistically significant.

\section{Sarcopenia and Treatment-Related Toxicities}

Grade 3-4 treatment-related toxicities are shown in Table 3. Patients with sarcopenia had significantly higher rates of grade 3 4 toxicities compared to those without sarcopenia $(36.2 \%$ vs $21.1 \%, p=0.034$ ). The main treatment-related toxicities of grade 3-4 were leukopenia (sarcopenic vs non-sarcopenic: 25 [26.6\%] vs 14 [15.6\%], $p=0.074$ ), neutropenia (19 [20.2\%] vs 8 [8.8\%], $p=0.037)$, esophagitis (13 [13.8\%] vs 10 [11.1\%], $p=0.828)$, and anorexia $(13[13.8 \%]$ vs 4 [4.4\%], $p=0.040)$.

TABLE 2 | Comparisons between patients with and without sarcopenia.

\begin{tabular}{|c|c|c|c|}
\hline Characteristics & $\begin{array}{c}\text { Sarcopenic } \\
n=94\end{array}$ & $\begin{array}{c}\text { Non-sarcopenic } \\
n=90\end{array}$ & $p$ \\
\hline Age & & & 0.166 \\
\hline$<70$ & $56(59.6 \%)$ & $63(70.0 \%)$ & \\
\hline$\geq 70$ & 38 (40.4\%) & 27 (30.0\%) & \\
\hline Sex & & & 0.384 \\
\hline Male & 75 (79.8\%) & $66(73.3 \%)$ & \\
\hline Female & $19(20.2 \%)$ & $24(26.7 \%)$ & \\
\hline KPS & & & 0.024 \\
\hline$\geq 80$ & $80(85.1 \%)$ & $86(95.6 \%)$ & \\
\hline 70 & $14(14.9 \%)$ & $4(4.4 \%)$ & \\
\hline Stage of primary tumor & & & 0.457 \\
\hline Stage I-II & $42(44.7 \%)$ & 35 (38.9\%) & \\
\hline Stage III & $52(55.3 \%)$ & $55(61.1 \%)$ & \\
\hline Recurrence pattern & & & 0.265 \\
\hline Anastomosis & $33(35.1 \%)$ & $24(26.7 \%)$ & \\
\hline Regional lymph node & $61(64.9 \%)$ & $66(73.3 \%)$ & \\
\hline Diabetes & & & 0.126 \\
\hline Yes & $12(12.8 \%)$ & $5(5.6 \%)$ & \\
\hline No & $82(87.2 \%)$ & 85 (94.4\%) & \\
\hline Weight loss in 6 months & & & 0.001 \\
\hline$\geq 5 \%$ & $44(46.8 \%)$ & $20(22.2 \%)$ & \\
\hline$<5 \%$ & $50(53.2 \%)$ & $70(77.8 \%)$ & \\
\hline BMI $\left(\mathrm{kg} / \mathrm{m}^{2}\right)$, mean $(\mathrm{SD})$ & $19.87 \pm 2.58$ & $21.15 \pm 2.86$ & $<0.001$ \\
\hline$<18.5$ & $25(26.6 \%)$ & $11(12.2 \%)$ & 0.016 \\
\hline$\geq 18.5$ & 69 (73.4\%) & 79 (87.8\%) & \\
\hline Albumin (g/L) & & & 0.033 \\
\hline$<35$ & 22 (23.4\%) & $10(11.1 \%)$ & \\
\hline$\geq 35$ & $72(76.6 \%)$ & $80(88.9 \%)$ & \\
\hline
\end{tabular}

KPS, karnofsky performance status; BMI, body mass index.
TABLE 3 | Comparisons of treatment-related toxicities between patients with and without sarcopenia.

\begin{tabular}{lccc}
\hline Toxicities & $\begin{array}{c}\text { Sarcopenic } \\
\mathbf{n = 9 4}\end{array}$ & $\begin{array}{c}\text { Non-sarcopenic } \\
\mathbf{n = 9 0}\end{array}$ & $\boldsymbol{p}$ \\
\hline Overall toxicity $\geq 3$ & $34(36.2 \%)$ & $19(21.1 \%)$ & 0.034 \\
Leukopenia & $25(26.6 \%)$ & $14(15.6 \%)$ & 0.074 \\
Neutropenia & $19(20.2 \%)$ & $8(8.8 \%)$ & 0.037 \\
Anemia & $7(7.4 \%)$ & $4(4.4 \%)$ & 0.537 \\
Thrombocytopenia & $4(4.3 \%)$ & $5(5.6 \%)$ & 0.743 \\
Esophagitis & $13(13.8 \%)$ & $10(11.1 \%)$ & 0.828 \\
Nausea/vomiting & $11(11.7 \%)$ & $8(8.9 \%)$ & 0.631 \\
Anorexia & $13(13.8 \%)$ & $4(4.4 \%)$ & 0.040 \\
\end{tabular}

With regard to treatment-related death, 2 patients (1 from trachea-esophageal fistula, and 1 from pulmonary embolism) died in the sarcopenic group versus 1 (gastrointestinal bleeding) in the non-sarcopenic group.

\section{Prognostic Significance of Sarcopenia in ESCC Patients}

As of May 18, 2020, median follow-up in this study was 11 months (range, 1-50 months), and 8 patients remained alive at the time of analysis. As observed in the Kaplan-Meier curve, patients with sarcopenia had worse survival compared to those without sarcopenia ( $p<0.001$, Figure 2$)$. Kaplan-Meier estimated 12 -month OS rate was $36.2 \%$ in the sarcopenic patients versus $57.8 \%$ in the non-sarcopenic patients. The 24 month OS rate for sarcopenic patients was 3.2\% compared to $17.8 \%$ for non-sarcopenic patients. In univariate analysis, KPS, the use of chemotherapy, weight loss in 6 months, BMI and sarcopenia were significantly associated with poor OS (Table 4).

Variables with $p<0.10$ in univariate analysis were included in the multivariate logistic regression analysis. In multivariate model, the presence of sarcopenia was the most significant independent prognostic factor of poor OS $(p=0.002)$ followed by worse KPS and advanced tumor stage (Table 4).

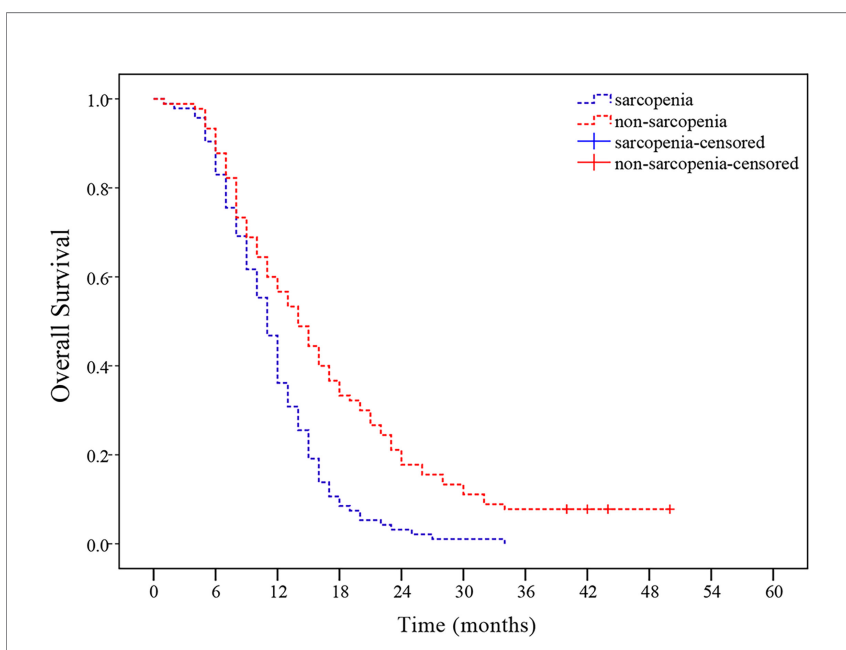

FIGURE 2 | Sarcopenia at presentation and overall survival (OS). Patients with sarcopenia had worse OS than those without sarcopenia $(p<0.001)$. 
TABLE 4 | Univariate and multivariate cox regression analysis for predictors of overall survival.

\begin{tabular}{|c|c|c|c|c|c|c|}
\hline Variable & \multicolumn{3}{|c|}{ Univariate analysis } & \multicolumn{3}{|c|}{ Multivariate analysis } \\
\hline \multicolumn{7}{|l|}{ Age (years) } \\
\hline$\geq 70$ & 0.788 & $0.577-1.076$ & 0.134 & & & \\
\hline \multicolumn{7}{|l|}{ Sex } \\
\hline Female & 1.00 & & & & & \\
\hline \multicolumn{7}{|l|}{ KPS } \\
\hline$\geq 80$ & 1.00 & & & & & \\
\hline 70 & 2.201 & $1.342-3.611$ & 0.002 & 2.331 & $1.296-4.190$ & 0.005 \\
\hline \multicolumn{7}{|l|}{ Primary Tumor location } \\
\hline Upper third & 1.00 & & & & & \\
\hline Middle third & 0.864 & $0.539-1.382$ & 0.541 & & & \\
\hline Lower third & 0.962 & $0.564-1.640$ & 0.887 & & & \\
\hline \multicolumn{7}{|l|}{ Stage of primary tumor } \\
\hline Anastomosis & 1.00 & & & & & \\
\hline Regional lymph node & 1.163 & $0.846-1.598$ & 0.353 & & & \\
\hline \multicolumn{7}{|l|}{ Concurrent chemotherapy } \\
\hline Yes & 1.00 & & & & & \\
\hline No & 1.674 & $1.044-2.683$ & 0.032 & 1.869 & $0.721-4.844$ & 0.198 \\
\hline \multicolumn{7}{|c|}{ Chemotherapeutic regimen } \\
\hline Docetaxel + cisplatin & 1.00 & & & & & \\
\hline S-1 & 0.833 & $0.588-1.182$ & 0.307 & & & \\
\hline \multicolumn{7}{|l|}{ Radiation dose (Gy) } \\
\hline$\geq 60$ & 1.00 & & & & & \\
\hline$<60$ & 0.768 & $0.546-1.081$ & 0.130 & & & \\
\hline \multicolumn{7}{|c|}{ Chemotherapy after radiotherapy } \\
\hline Yes & 1.00 & & & & & \\
\hline$<35$ & 0.993 & $0.669-1.472$ & 0.970 & & & \\
\hline \multicolumn{7}{|l|}{ SMI } \\
\hline Non-sarcopenic & 1.00 & & & & & \\
\hline Sarcopenic & 1.907 & $1.397-2.602$ & $<0.001$ & 1.729 & $1.231-2.428$ & 0.002 \\
\hline
\end{tabular}

HR, hazard ratio; Cl, confidence interval; KPS, karnofsky performance status; BMI, body mass index; SMI, skeletal muscle index.

\section{DISCUSSION}

It is well established that sarcopenia is a significant factor of poor survival across various cancer types (20-22). However, to our knowledge, there are no reports discussing the relationship between sarcopenia and survival in postoperative locoregional recurrent ESCC patients. Thus, in the present study, we first investigated the incidence of sarcopenia in 184 patients with postoperative locoregional recurrent ESCC and then evaluated associations with treatment toxicity and survival. Our results confirmed that over $50 \%$ of patients had sarcopenia. These patients were more likely to present grade $\geq 3$ toxicities compared with non-sarcopenic patients. In addition, the multivariable analysis showed that sarcopenia was a significant independent prognostic factor for poor survival.

The prevalence of sarcopenia in patients with ESCC fluctuates significantly, with reports ranging from $16 \%$ to $75 \%$ (23). However, these studies vary in the definition of sarcopenia, tumor stage and histological type. In this study, inclusion criteria were limited to patient with postoperative locoregional recurrent diseases, and $51.1 \%(94 / 184)$ of patients had sarcopenia at presentation, which is higher than a prior study (16\%) involving patients with locally advanced EC (9). However, because the majority of patients $(81.2 \%)$ in that study were esophageal adenocarcinoma and $43 \%$ was visceral obesity, direct comparison with the current study is difficult. To date, 
the optimal cut-off values chosen to diagnose sarcopenia remain a matter of debate. In western countries, the sex-specific cut-off values for L3 SMI $\left(52.4 / \mathrm{cm}^{2} / \mathrm{m}^{2}\right.$ for men and $38.5 / \mathrm{cm}^{2} / \mathrm{m}^{2}$ for women) proposed by Prado et al. are most commonly used to evaluate sarcopenia in patients with cancer (24). Using the Prado's criteria, the population of sarcopenia in the present study would increase to 129 (70.1\%), which was consistent with that in Siegal SR study in patients with EC (25). However, this criteria might not be applicable to Chinese ESCC patients because the BMI differs considerably between Asian and western populations. In the present population, we diagnosed sarcopenia according to the cut-off values proposed by Nakashima Y (18). As the population of that study is very similar to our population. In ESCC, the nutritional impairment due to dysphagia, pain, systemic inflammation and increased metabolic rate may have promoted the development of sarcopenia.

Several studies have reported that patients with sarcopenia had higher rates of treatment-related toxicity in various malignancies (26). In a study on patients with metastatic breast cancer, chemotherapy toxicity was more commonly observed among sarcopenic patients (27).. In locally advanced EC patients treated with neoadjuvant radiochemotherapy, Panje CM et al. showed that the incidence of grade $\geq 3$ toxicity was significantly higher in sarcopenic compared with non-sarcopenic patients (28). In EC patients treated by esophagectomy, Ida $\mathrm{S}$ et al. revealed that sarcopenia was closely associated with higher rates of respiratory complications (29). In our study, patients with sarcopenia had greater risk of grade 3-4 CRT-related toxicity, so we believe that the clinical management of patients with sarcopenia before CRT, such as physical exercise, nutrition management, as well as pharmacologic treatment, could prevent toxicity.

Sarcopenia has been correlated with shorter survival in certain solid cancers such as oropharyngeal squamous cell carcinoma, non-small cell lung cancer, and nasopharyngeal carcinoma (30-32). Currently, to our knowledge, 6 studies investigated the effect of sarcopenia on survival in EC patients who received surgical resection $(9,18,33-36)$. Three of these studies reported that sarcopenia was significantly associated with poor survival. However, the impact of sarcopenia on survival after CRT has not been clearly established in patients with postoperative locoregional recurrent ESCC. In the current study, we found that the 12-month and 24-month OS were significantly lower for patients with sarcopenia compared to those without, which is consistent with 2 recent studies on patients with unresectable advanced EC treated with CRT (37, 38). In contrast, in another study of 300 patients who treated with trimodality therapy, sarcopenia was not associated with poor OS in a subset of 61 patients who underwent neoadjuvant radiochemotherapy (28). This may be because the numbers of patients were relatively small and the cut-off values used for sarcopenia were looser than ours. Furthermore, differences in treatments and stage of disease may also affect the results.

Our study has several limitations. Firstly, the conclusions are drawn from a sing-institute retrospective analysis and the sample size was small. Secondly, given its retrospective design, evaluation of muscle strength and physical activity were not available. Thirdly, a small proportion of patients $(21.7 \%)$ received anlotinib-targeted therapy or PD-1 inhibitor after tumor progression. This may have affected our results. Thus, further multi-institutional prospective clinical trials are needed to confirm our results.

In conclusion, our results show that sarcopenia is significantly associated with treatment-related toxicity and poorer outcomes in postoperative locoregional recurrent ESCC patients receiving CRT.

\section{DATA AVAILABILITY STATEMENT}

The original contributions presented in the study are included in the article/supplementary material. Further inquiries can be directed to the corresponding authors.

\section{ETHICS STATEMENT}

The studies involving human participants were reviewed and approved by Huai'an First Hospital. Written informed consent for participation was not required for this study in accordance with the national legislation and the institutional requirements.

\section{AUTHOR CONTRIBUTIONS}

Y-YX, X-LZ, W-GZ, and YT conceived and designed the experiments and were responsible for data analysis and writing the manuscript. C-HY, W-WW, and F-ZJ were responsible for providing the clinical samples. $\mathrm{D}-\mathrm{CH}$ was responsible for data collection. All authors contributed to the article and approved the submitted version.

\section{FUNDING}

This work was supported by grants from National Nature Science Foundation of China (Grant No. 82002536) and Huai'an Natural Science Research Project (HAB201930). These funding agencies were not involved in the study design, the collection, analysis and interpretation of data, writing the report, and the decision to submit the article for publication.

\section{ACKNOWLEDGMENTS}

The authors acknowledge Juan Tan for his professional statistical analysis. 


\section{REFERENCES}

1. Smyth EC, Lagergren J, Fitzgerald RC, Lordick F, Shah MA, Lagergren P, et al. Oesophageal Cancer. Nat Rev Dis Primers (2017) 3:17048. doi: 10.1038/ nrdp. 2017.48

2. Yang H, Liu H, Chen Y, Zhu C, Fang W, Yu Z, et al. Neoadjuvant Chemoradiotherapy Followed by Surgery Versus Surgery Alone for Locally Advanced Squamous Cell Carcinoma of the Esophagus (NEOCRTEC5010): A Phase III Multicenter, Randomized, Open-Label Clinical Trial. J Clin Oncol (2018) 36:2796-803. doi: 10.1200/JCO.2018.79.1483

3. Herskovic A, Russell W, Liptay M, Fidler MJ, Al-Sarraf M. Esophageal Carcinoma Advances in Treatment Results for Locally Advanced Disease: Review. Ann Oncol (2012) 23:1095-103. doi: 10.1093/annonc/mdr433

4. Honing J, Smit JK, Muijs CT, Burgerhof JGM, de Groot JW, Paardekooper G, et al. A Comparison of Carboplatin and Paclitaxel With Cisplatinum and 5Fluorouracil in Definitive Chemoradiation in Esophageal Cancer Patients. Ann Oncol (2014) 25:638-43. doi: 10.1093/annonc/mdt589

5. Chen J, Yin W, Yao H, Gu W. Salvage Treatment for Lymph Node Recurrence After Radical Resection of Esophageal Squamous Cell Carcinoma. Radiat Oncol (2019) 14:169. doi: 10.1186/s13014-019-1377-y

6. Pennathur A, Gibson MK, Jobe BA, Luketich JD. Oesophageal Carcinoma. Lancet (2013) 381:400-12. doi: 10.1016/S0140-6736(12)60643-6

7. Baumgartner RN, Koehler KM, Gallagher D, Romero L, Heymsfield SB, Ross RR, et al. Epidemiology of Sarcopenia Among the Elderly in New Mexico. Am J Epidemiol (1998) 147:755-63. doi: 10.1093/oxfordjournals.aje.a009520

8. Cruz-Jentoft AJ, Baeyens JP, Bauer JM, Boirie Y, Cederholm T, Landi F, et al. Sarcopenia: European Consensus on Definition and Diagnosis: Report of the European Working Group on Sarcopenia in Older People. Age Ageing (2010) 39:412-23. doi: 10.1093/ageing/afq034

9. Elliott JA, Doyle SL, Murphy CF, King S, Guinan EM, Beddy P, et al. Sarcopenia: Prevalence, and Impact on Operative and Oncologic Outcomes in the Multimodal Management of Locally Advanced Esophageal Cancer. Ann Surg (2017) 266:822-30. doi: 10.1097/SLA.0000000000002398

10. Anandavadivelan P, Brismar TB, Nilsson M, Johar AM, Martin L. Sarcopenic Obesity: A Probable Risk Factor for Dose Limiting Toxicity During NeoAdjuvant Chemotherapy in Oesophageal Cancer Patients. Clin Nutr (2016) 35:724-30. doi: 10.1016/j.clnu.2015.05.011

11. Cruz-Jentoft AJ, Bahat G, Bauer J, Boirie Y, Bruyere O, Cederholm T, et al. Sarcopenia: Revised European Consensus on Definition and Diagnosis. Age Ageing (2019) 48:16-31. doi: 10.1093/ageing/afy169

12. Shachar SS, Williams GR, Muss HB, Nishijima TF. Prognostic Value of Sarcopenia in Adults With Solid Tumours: A Meta-Analysis and Systematic Review. Eur J Cancer (2016) 57:58-67. doi: 10.1016/j.ejca.2015.12.030

13. Cho Y, Kim JW, Keum KC, Lee CG, Jeung HC, Lee IJ. Prognostic Significance of Sarcopenia With Inflammation in Patients With Head and Neck Cancer Who Underwent Definitive Chemoradiotherapy. Front Oncol (2018) 8:457. doi: $10.3389 /$ fonc.2018.00457

14. Lee BM, Cho Y, Kim JW, Jeung HC, Lee IJ. Prognostic Significance of Sarcopenia in Advanced Biliary Tract Cancer Patients. Front Oncol (2020) 10:1581. doi: 10.3389/fonc.2020.01581

15. Ozawa Y, Nakano T, Taniyama Y, Sakurai T, Onodera Y, Kamiya K, et al. Evaluation of the Impact of Psoas Muscle Index, a Parameter of Sarcopenia, in Patients With Esophageal Squamous Cell Carcinoma Receiving Neoadjuvant Therapy. Esophagus (2019) 16:345-51. doi: 10.1007/s10388-019-00670-3

16. Dijksterhuis WPM, Pruijt MJ, van der Woude SO, Klaassen R, Kurk SA, van Oijen MGH, et al. Association Between Body Composition, Survival, and Toxicity in Advanced Esophagogastric Cancer Patients Receiving Palliative Chemotherapy. J Cachexia Sarcopenia Muscle (2019) 10:199-206. doi: $10.1002 / \mathrm{jcsm} .12371$

17. Mourtzakis M, Prado CM, Lieffers JR, Reiman T, McCargar LJ, Baracos VE. A Practical and Precise Approach to Quantification of Body Composition in Cancer Patients Using Computed Tomography Images Acquired During Routine Care. Appl Physiol Nutr Metab (2008) 33:997-1006. doi: 10.1139/ H08-075

18. Nakashima Y, Saeki H, Nakanishi R, Sugiyama M, Kurashige J, Oki E, et al. Assessment of Sarcopenia as a Predictor of Poor Outcomes After Esophagectomy in Elderly Patients With Esophageal Cancer. Annf Surg (2018) 267:1100-4. doi: 10.1097/SLA.0000000000002252
19. WHO expert consultation. Appropriate body-mass index for Asian populations and its implications for policy and intervention strategies. Lancet (2004) 363:157-63. doi :10.1016/S0140-6736(03)15268-3

20. Iritani S, Imai K, Takai K, Hanai T, Ideta T, Miyazaki T, et al. Skeletal Muscle Depletion is an Independent Prognostic Factor for Hepatocellular Carcinoma. J Gastroenterol (2015) 50:323-32. doi: 10.1007/s00535-014-0964-9

21. Grossberg AJ, Chamchod S, Fuller CD, Mohamed AS, Heukelom J, Eichelberger H, et al. Association of Body Composition With Survival and Locoregional Control of Radiotherapy-Treated Head and Neck Squamous Cell Carcinoma. JAMA Oncol (2016) 2:782-9. doi: 10.1001/jamaoncol. 2015.6339

22. Feliciano EMC, Kroenke CH, Meyerhardt JA, Prado CM, Bradshaw PT, Kwan ML, et al. Association of Systemic Inflammation and Sarcopenia With Survival in Nonmetastatic Colorectal Cancer: Results From the C SCANS Study. JAMA Oncol (2017) 3:e172319. doi: 10.1001/jamaoncol.2017.2319

23. Boshier PR, Heneghan R, Markar SR, Baracos VE, Low DE. Assessment of Body Composition and Sarcopenia in Patients With Esophageal Cancer: A Systematic Review and Meta-Analysis. Dis Esophagus (2018) 31:1-11. doi: $10.1093 /$ dote/doy047

24. Prado CMM, Lieffers JR, McCargar LJ, Reiman T, Sawyer MB, Martin L, et al. Prevalence and Clinical Implications of Sarcopenic Obesity in Patients With Solid Tumours of the Respiratory and Gastrointestinal Tracts: A PopulationBased Study. Lancet Oncol (2008) 9:629-35. doi: 10.1016/S1470-2045(08) 70153-0

25. Siegal SR, Dolan JP, Dewey EN, Guimaraes AR, Tieu BH, Schipper PH, et al. Sarcopenia is Not Associated With Morbidity, Mortality, or Recurrence After Esophagectomy for Cancer. Am J Surg (2018) 215:813-7. doi: 10.1016/ j.amjsurg.2017.12.017

26. Tsai S. Importance of Lean Body Mass in the Oncologic Patient. Nutr Clin Pract (2012) 27:593-8. doi: 10.1177/0884533612457949

27. Prado CMM, Baracos VE, McCargar LJ, Reiman T, Mourtzakis M, Tonkin K, et al. Sarcopenia as a Determinant of Chemotherapy Toxicity and Time to Tumor Progression in Metastatic Breast Cancer Patients Receiving Capecitabine Treatment. Clin Cancer Res (2009) 15:2920-6. doi: 10.1158/ 1078-0432.CCR-08-2242

28. Panje CM, Höng L, Hayoz S, Baracos VE, Herrmann E, Garcia Schüler H,M, et al. Skeletal Muscle Mass Correlates With Increased Toxicity During Neoadjuvant Radiochemotherapy in Locally Advanced Esophageal Cancer: A SAKK 75/08 Substudy. Radiat Oncol (2019) 14:166. doi: 10.1186/s13014019-1372-3

29. Ida S, Watanabe M, Yoshida N, Baba Y, Umezaki N, Harada K, et al. Sarcopenia is a Predictor of Postoperative Respiratory Complications in Patients With Esophageal Cancer. Ann Surg Oncol (2015) 22:4432-7. doi: 10.1245/s10434-015-4559-3

30. Olson B, Edwards J, Stone L, Jiang A, Zhu X, Holland J, et al. Association of Sarcopenia With Oncologic Outcomes of Primary Surgery or Definitive Radiotherapy Among Patients With Localized Oropharyngeal Squamous Cell Carcinoma. JAMA Otolaryngol Head Neck Surg (2020) 146:714. doi: 10.1001/jamaoto.2020.1154

31. Nakamura R, Inage Y, Tobita R, Yoneyama S, Numata T, Ota K, et al. Sarcopenia in Resected NSCLC: Effect on Postoperative Outcomes. J. Thorac Oncol (2018) 13:895-903. doi: 10.1016/j.jtho.2018.04.035

32. Hua X, Liao J-F, Huang X, Huang H-Y, Wen W, Long Z-Q, et al. Sarcopenia is Associated With Higher Toxicity and Poor Prognosis of Nasopharyngeal Carcinoma. Ther Adv Med Oncol (2020) 12:175883592094761. doi: 10.1177/ 17588359

33. Harada K, Ida S, Baba Y, Ishimoto T, Kosumi K, Tokunaga R, et al. Prognostic and Clinical Impact of Sarcopenia in Esophageal Squamous Cell Carcinoma. Dis Esophagus (2016) 29:627-33. doi: 10.1111/dote.12381

34. Grotenhuis BA, Shapiro J, van Adrichem S, de Vries M, Koek M, Wijnhoven BP, et al. Sarcopenia/Muscle Mass is Not a Prognostic Factor for Short- and Long-Term Outcome After Esophagectomy for Cancer. World J Surg (2016) 40:2698-704. doi: 10.1007/s00268-016-3603-1

35. Srpcic M, Jordan T, Popuri K, Sok M. Sarcopenia and Myosteatosis at Presentation Adversely Affect Survival After Esophagectomy for Esophageal Cancer. Radiol Oncol (2020) 54:237-46. doi: 10.2478/raon-2020-0016

36. Tamandl D, Paireder M, Asari R, Baltzer PA, Schoppmann SF, Ba-Ssalamah A. Markers of Sarcopenia Quantified by Computed Tomography Predict 
Adverse Long-Term Outcome in Patients With Resected Oesophageal or Gastro-Oesophageal Junction Cancer. Eur Radiol (2016) 26:1359-67. doi: 10.1007/s00330-015-3963-1

37. Sato S, Kunisaki C, Suematsu H, Tanaka Y, Miyamoto H, Kosaka T, et al. Impact of Sarcopenia in Patients With Unresectable Locally Advanced Esophageal Cancer Receiving Chemoradiotherapy. Vivo (2018) 32:603-10. doi: 10.21873 /invivo.11282

38. Onishi S, Tajika M, Tanaka T, Hirayama Y, Hara K, Mizuno N, et al. Prognostic Significance of Sarcopenia in Patients With Unresectable Advanced Esophageal Cancer. J Clin Med (2019) 8:1-12. doi: 10.3390/ jcm8101647
Conflict of Interest: The authors declare that the research was conducted in the absence of any commercial or financial relationships that could be construed as a potential conflict of interest.

Copyright $\odot 2021 \mathrm{Xu}, \mathrm{Zhou}, \mathrm{Yu}$, Wang, Ji, He, Zhu and Tong. This is an open-access article distributed under the terms of the Creative Commons Attribution License (CC BY). The use, distribution or reproduction in other forums is permitted, provided the original author(s) and the copyright owner(s) are credited and that the original publication in this journal is cited, in accordance with accepted academic practice. No use, distribution or reproduction is permitted which does not comply with these terms. 